\title{
Piecewise-Linear Constrained Control for Timed Continuous Petri Nets
}

\author{
C. Renato Vázquez
}

constraints can be found in the literature, it has been found difficult to apply those to $T C P N$ systems. Thus there exists necessity for searching more control techniques for this particular model. For instance, classic works ([4] , [5]) deal with controllability and stabilization problems for systems with bounded inputs, however, in those works the input can take negative values, which is prohibitive in our model. In [6] the controllability on linear systems with nonnegative inputs is addressed. Regarding to $P W L$ systems, in [7] a problem concerning production systems is formulated as a $H_{\infty}$ control problem for a piecewise affine system; there, an optimization program subject to LMI constraints is proposed. In [8], the control design problem for $P W L$ systems is studied through quadratic Lyapunov functions and convex optimization. However, in [9] it was shown that TCPN systems are not controllable in the classical sense, therefore, such concepts and techniques cannot be directly applied to our model. Despite such result, in [10] it was proved that if some conditions are fulfilled then there exists a set of interesting points (the set of possible stationary operating points of the model) in which the system exhibits the controllability property, i.e. any state of such set is reachable from any other one in it. In order to study such property, a local controllability concept was proposed for $T C P N$ systems, and sufficient and necessary conditions for controllability were introduced.

An interesting approach for the Control synthesis on Piecewise-Affine systems was provided in [11]. For the systems considered in such work, the continuous state evolves as an affine linear system inside simpleces (polytopes of full dimension with a minimum number of vertices), associated to discrete sates. The control problem addressed in those papers consists in the synthesis of an affine linear control law, for each of the simpleces, that ensures that the continuous state does not leave the current simplex by crossing undesirable "facets", which at the underlying discrete system means blocking undesirable events. The solution proposed is based on directing the field vector, of the closed-loop system, towards suitable directions (inside or outside to the simplex, depending the case) at the vertices of the simplex. Even when $T C P N$ systems can be viewed as such piecewiseaffine systems, there exist two major problems for applying the results introduced in that work. The first is that the input in $T C P N$ is not constrained to a polytope, but it is upper bounded by a function of the state. The second problem, and most important, is that in $T C P N$ systems it is very frequent to find "polytopes" having vertices at which the input must be null, becoming impossible to direct the field vector in the required direction (a simple example is provided in Section

\footnotetext{
The research leading to these results has received funding from the European Community's Seventh Framework Programme (FP7/2007-2013) under grant agreement no 224498.

This work was partially supported by projects CICYT and FEDER DPI2006-15390.

Authors are with Dep. de Informática e Ingeniería de Sistemas, Centro Politécnico Superior, Universidad de Zaragoza, E-50018 Zaragoza, Spain $\{$ cvazquez, silva\}@unizar.es

This paper has been written in honor to Prof. L. Recalde, who passed away last December.
}

\author{
Manuel Silva
}

\begin{abstract}
Timed Continuous Petri Net (TCPN) systems are
piecewise linear models with input constraints that can ap-
\end{abstract} variables)

Even if a lot of research related to controllability and 
4), then it is necessary to consider different approaches for controlling continuous net systems.

Taking advantage of the particular estructure of $T C P N$, control laws for these systems have been proposed by using different techniques. In [12] a solution based on Model Predictive Control was proposed, finding computational complexity problems for the explicit approach that make its application prohibitive. In [13] a tracking control approach was introduced, considering step and ramp references and low-andhigh gain controllers. Local stability and input boundedness were proved for a class of PNs. In those papers all transitions are assumed to be controllable. Uncontrollable transitions were considered in [14], where a gradient-based controller was proposed for driving the output towards the desired value. However, since the input constraints are not considered for the minimization of the cost function, convergence is not guaranteed.

In this paper, uncontrollable transitions are considered. In order to reduce computational complexity problems and to provide feasibility and effectiveness, we propose in this case a classical approach by adapting the well-known linear feedback control structure. Since the system is piecewise linear, for each linear mode (corresponding to a particular configuration) a stabilizing gain matrix is considered. These can be computed by using any pole assignment technique. In order to guarantee the boundedness of the input, suitable intermediate target markings (states) are computed by solving on-line a LPP, obtaining thus a piecewise-linear constrained control structure. Convergence to the required marking and boundedness of the input are demonstrated, whenever the system fulfills some controllability conditions introduced in [10].

This paper is organized as follows: in Section 2 an overview of continuous and TCPN is presented, while in Section 3, some previous results related to controllability are recalled. In Section 4 the case in which the initial and desired markings belong to the same region is considered, and next, in Section 5, the case with different regions is studied. Conclusions are presented in Section 6 .

\section{BASIC CONCEPTS}

The structure $\mathcal{N}=\langle P, T$, Pre, Post $\rangle$ of continuous $P N$ is the same as the structure of discrete PN, i.e. $P$ and $T$ are finite disjoint sets of places and transitions, respectively, Pre and Post are $|P| \times|T|$ sized, natural valued, pre- and post-incidence matrices.

The main difference is in the evolution rule, since in continuous PN firing is not restricted to integer amounts, and so the marking $\mathbf{m}$ is not forced to be integer. More precisely, a transition $t$ is enabled at $\mathbf{m}$ iff for every $p \in$ ${ }^{\bullet} t, \mathbf{m}[p]>0$, and its enabling degree is enab $(t, \mathbf{m})=$ $\min _{p \in \bullet}\{\mathbf{m}[p] /$ Pre $[p, t]\}$. The firing of $t$ in a certain amount $\alpha \leq \operatorname{enab}(t, \mathbf{m})$ leads to a new marking $\mathbf{m}^{\prime}=\mathbf{m}+\alpha \cdot \mathbf{C}[P, t]$, where $\mathbf{C}=$ Post - Pre is the token-flow matrix.

Right and left rational annulers of $\mathbf{C}$ are called $T$ - and $P$-flows, respectively. If there exists $\mathbf{y}>\mathbf{0}(\mathbf{x}>0)$ s.t. $\mathbf{y C}=\mathbf{0}(\mathbf{C x}=\mathbf{0})$, the net is said to be conservative (consistent). For reachability, the limit concept is used, and a marking reached in the limit of an infinitely long sequence is considered reachable [15].

A Timed Continuous Petri Net (TCPN) is a continuous PN with a vector $\lambda \in \mathbb{R}_{>0}^{|T|}$. Here, infinite server semantics is considered (sometimes called variable speed, see [2] for an introduction to semantics used in TCPN), thus the flow through a timed transition $t$ is the product of the rate $\boldsymbol{\lambda}[t]$ and $\operatorname{enab}(t, \mathbf{m})$, the instantaneous enabling representing the number of active serves, i.e., $\mathbf{f}(\mathbf{m})[t]=\boldsymbol{\lambda}[t] \cdot \operatorname{enab}(t, \mathbf{m})=$ $\boldsymbol{\lambda}[t] \cdot \min _{p \in \bullet}\{\mathbf{m}[p] / \operatorname{Pre}[p, t]\}$. For the flow to be well defined we will assume that $\forall t \in T,|\bullet t| \geq 1$. The "min" in the definition leads to the concept of configurations: a configuration assigns to each transition one place that for some markings will control its firing rate (i.e. it is constraining that transition). The number of configurations is upper bounded by $\left.\prod_{t \in T}\right|^{\bullet} t \mid$.

The flow through the transitions can be written in a vectorial form as $\mathbf{f}(\mathbf{m})=\Lambda \Pi(\mathbf{m}) \mathbf{m}$, where $\Lambda$ is a diagonal matrix whose elements are those of $\lambda$, and $\Pi(\mathbf{m})$ is the configuration operator matrix at $\mathbf{m}$, defined by elements as

$$
\boldsymbol{\Pi}(\mathbf{m})[i, j]=\left\{\begin{array}{cc}
\frac{1}{\operatorname{Pre}\left[p_{j}, t_{i}\right]} & \text { if } p_{j} \text { is constraining } t_{i} \\
0 & \text { otherwise }
\end{array}\right.
$$

If more than one place is constraining the flow of a transition at a given marking, any of them can be used, but only one is taken.

Control action may only be a reduction of the flow through the transitions. That is, transitions (machines for example) cannot work faster than their nominal speed. Transitions in which a control action can be applied are called controllable. The effective flow through a transition which is being controlled can be represented as: $\mathbf{w}_{i}(\tau)=\boldsymbol{\lambda}\left[t_{i}\right] \cdot \operatorname{enab}(\tau)\left[t_{i}\right]-$ $u(\tau)\left[t_{i}\right]$, where $0 \leq u(\tau)\left[t_{i}\right] \leq \lambda\left(t_{i}\right) \cdot \operatorname{enab}(\tau)\left[t_{i}\right]$. The control vector $\mathbf{u} \in \mathbb{R}^{|T|}$ is defined s.t. $\mathbf{u}_{i}$ represents the control action on $t_{i}$. If $t_{i}$ is not controllable then $\mathbf{u}_{i}=0$. The forced flow vector is expressed as $\mathbf{w}(\mathbf{m}, \mathbf{u})=\Lambda \Pi(\mathbf{m}) \mathbf{m}-$ u. The set of all controllable transitions is denoted by $T_{c}$, and the set of uncontrollable transitions is $T_{n c}=T-T_{c}$.

The behavior of a TCPN forced system is described by the state equation:

$$
\begin{gathered}
\stackrel{\bullet}{\mathbf{m}}=\mathrm{C} \Lambda \Pi(\mathbf{m}) \mathbf{m}-\mathbf{C u} \\
\mathbf{0} \leq \mathrm{u} \leq \Lambda \Pi(\mathbf{m}) \mathbf{m}
\end{gathered}
$$

A control action that fulfills the required constraints, i.e. $\forall t_{i} \in T_{n c} \mathbf{u}_{i}=0$ and $\mathbf{0} \leq \mathbf{u} \leq \boldsymbol{\Lambda} \Pi(\mathbf{m}) \mathbf{m}$, is called suitable bounded (s.b.). If an input is not s.b. then it cannot be applied. A marking $\mathbf{m}$ for which $\exists \mathbf{u}$ s.b. at $\mathbf{m}$ such that $\dot{\mathbf{m}}=\mathbf{C}[\boldsymbol{\Lambda} \boldsymbol{\Pi}(\mathbf{m}) \mathbf{m}-\mathbf{u}]=\mathbf{0}$ is called equilibrium marking, and $\mathbf{u}$ and $\mathbf{w}(\mathbf{m}, \mathbf{u})$ are said to be its equilibrium input and flow, respectively.

Marking $\mathbf{m}_{2}$ is said to be reachable from $\mathbf{m}_{1}$ if $\exists \mathbf{u}$ s.b. that transfers the system from $\mathbf{m}_{1}$ to $\mathbf{m}_{2}$ in either finite or infinite time (lim-reachable). If $\mathbf{m}_{1}$ is the initial marking then $\mathbf{m}_{2}$ is simply called reachable. The set of reachable markings can be defined for autonomous continuous PN and TCPN 
systems [15]. In the sequel, the term reachability always refers to timed systems.

\section{Controllability}

In this section, some controllability concepts are recalled from [10].

If $\mathbf{y}$ is a $P$-flow, then for any reachable marking $\mathbf{m}$, $\mathbf{y}^{T} \mathbf{m}=\mathbf{y}^{T} \mathbf{m}_{\mathbf{0}}$. So, whenever a TCPN system has $P$ flows, linear dependencies between marking variables appear, introducing token conservation laws, a class of state invariants. Therefore, systems with P-flows are not controllable in the classical sense. However, we are interested in the controllability "over" this invariant. In the sequel, this state invariant will be denoted as Class $\left(\mathbf{m}_{\mathbf{0}}\right)$. Notice that every reachable marking belongs to Class $\left(\mathbf{m}_{\mathbf{0}}\right)$, but the reverse is not true for timed models. This set is divided into subsets of markings associated to the same configuration, which are named regions. Then, for each particular configuration matrix $\Pi_{i}$ there is its corresponding region $\Re_{i}=\{\mathbf{m} \in$ $\left.\operatorname{Class}\left(\mathbf{m}_{\mathbf{0}}\right) \mid \boldsymbol{\Pi}(\mathbf{m})=\boldsymbol{\Pi}_{i}\right\}$. The Class $\left(\mathbf{m}_{\mathbf{0}}\right)$ and all the regions are convex sets. Inside each region the state equation (1) is linear $(\boldsymbol{\Pi}(\mathbf{m})$ is constant). Notice that configurations (sets of arcs that constraint transitions) are defined at net level, configuration matrices at the algebraic level and regions at the state space level. In the sequel, let us denote by $\operatorname{int}\left(\operatorname{Class}\left(\mathbf{m}_{\mathbf{0}}\right)\right)$ and $\operatorname{int}\left(\Re_{i}\right)$ the sets of interior markings of Class $\left(\mathbf{m}_{\mathbf{0}}\right)$ and $\Re_{i}$, respectively (considering the space generated by the columns of $\mathbf{C}$ ).

Now, let us remark that the classical controllability definition cannot be applied to TCPN systems because the set of reachable markings (a subset of Class $\left(\mathbf{m}_{\mathbf{0}}\right)$ ) never compose a vector space, and also the input must be suitable bounded (i.e. $\mathbf{0} \leq \mathbf{u} \leq \mathbf{\Lambda} \Pi(\mathbf{m}) \mathbf{m})$. Therefore, an adaptation of the classical controllability definition was proposed in [10].

Definition 1: The TCPN system $\left\langle\mathcal{N}, \boldsymbol{\lambda}, \mathbf{m}_{\mathbf{0}}\right\rangle$ is controllable with bounded input (BIC) over $S \subseteq \operatorname{Class}\left(\mathbf{m}_{\mathbf{0}}\right)$ if for any two markings $\mathbf{m}_{1}, \mathbf{m}_{2} \in S$ there exists an input $\mathbf{u}$ that transfers the system from $\mathbf{m}_{1}$ to $\mathbf{m}_{2}$ in finite or infinite time, and it is suitable bounded along the marking trajectory.

The controllability in TCPN systems is studied over sets of equilibrium markings because they represent "the stationary operating points" of the modeled system. The set of all equilibrium markings is defined as: $E_{q} S=\{m \in$ $\operatorname{Class}\left(\mathbf{m}_{\mathbf{0}}\right) \mid \exists \mathbf{u}$ s.b. at $\mathbf{m}$ s.t. $\left.\mathbf{C}(\mathbf{\Lambda} \Pi(\mathbf{m}) \mathbf{m}-\mathbf{u})=0\right\}$.

The set of all equilibrium markings in the i-th region is defined as $E_{i}=\left\{\mathbf{m} \mid \mathbf{m} \in E_{q} S \cap \Re_{i}\right\}$. Since the system is linear inside each region, the controllability is linearly studied first over each $E_{i}$. Now, let us introduce some useful definitions related to $E_{i}$.

Definition 2: A Generator of $E_{i} \neq \varnothing$ is a kind of basis for it, formally defined as a full column rank matrix $\mathbf{G}_{i}$ that fulfills:

a) $\forall \mathbf{m}_{1}, \mathbf{m}_{2} \in E_{i},\left(\mathbf{m}_{1}-\mathbf{m}_{2}\right)$ is in the range of $\mathbf{G}_{i}$.

b) $\mathbf{G}_{i}$ is minimal (if one of its columns is removed then $\mathbf{a}$ is false).

Definition 3: A controllable transition $t_{j}$ is said to be fully controllable at $E_{i}$ if there exists an equilibrium mark-

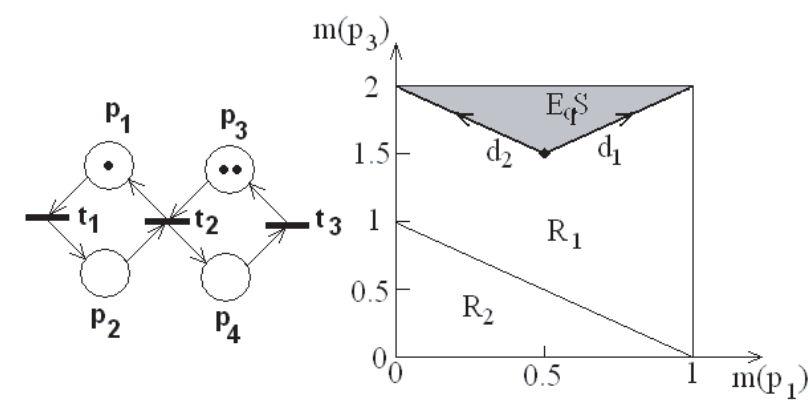

Fig. 1. The set $E_{q} S$, and subsets $E_{i}$ and $E_{i}^{+}$.

ing $\mathbf{m}^{q} \in E_{i}$ with an equilibrium input $\mathbf{u}^{q}$ such that $\left[\boldsymbol{\Lambda} \boldsymbol{\Pi}_{i} \mathbf{m}^{q}\right]_{j}>\mathbf{u}_{j}^{q}>0$. In other case, $t_{j}$ is said to be partially controllable. The set of fully (partially) controllable transitions at $E_{i}$ is denoted as $T_{c f}^{i}\left(T_{c p}^{i}\right)$.

Definition 4: The subset of $E_{i}$, in which the equilibrium flow can be positive, is defined as

$$
E_{i}^{+}=\left\{\mathbf{m}^{q} \in E_{i} \mid \exists \mathbf{u}^{q} \text { such that } \mathbf{w}^{q}>0\right\}
$$

In order to clarify the sense of these definitions, see the following example.

Example 1: Consider the system of fig. 1 with $\boldsymbol{\Lambda}=\mathbf{I}$ and $T_{c}=\left\{t_{1}, t_{2}\right\}$. There exist two possible configurations: that in which $t_{2}$ is constrained by $p_{2}$ (related to $\Re_{1}$ ) and the other in which $t_{2}$ is constrained by $p_{3}$ (related to $\Re_{2}$ ). Fig. 1 also shows the Class $\left(\mathbf{m}_{0}\right)$ (the marking of $p_{1}$ and $p_{3}$ is enough to determine a reachable marking, since this net has two P-semiflows). The whole grey triangle corresponds to $E_{1}$. Actually, in this example $E_{1}=E_{q} S$ and $E_{2}=\varnothing$. A Generator of $E_{1}$ is given by

$$
\mathbf{G}_{1}=\left[\begin{array}{rrrr}
1, & -1, & 1, & -1 \\
-1, & 1, & 1, & -1
\end{array}\right]^{T}
$$

The column vectors of $\mathbf{G}_{1}$, scaled and restricted to $p_{1}$ and $p_{3}$, are represented by $\mathbf{d}_{1}$ and $\mathbf{d}_{2}$ in fig. 1 . The triangle without the upper edge (that in which $m\left(p_{3}\right)=2$ ) corresponds to $E_{1}^{+}$. Furthermore, since for any marking in the interior of the triangle its equilibrium input is positive at the entries related to the controllable transitions, $T_{c f}^{1}=T_{c}$ and $T_{c p}^{1}=\varnothing$.

In the sequel, let us denote as $\mathbf{C}_{c}$ and $\mathbf{C}_{c f}^{i}$ the matrices built with the columns of $\mathbf{C}$ related to the transitions in $T_{c}$ and $T_{c f}^{i}$, respectively. Next corollary, proved in [10], introduces conditions for controllability over a given $E_{i}^{+}$.

Corollary 1: Let $\left\langle\mathcal{N}, \boldsymbol{\lambda}, \mathbf{m}_{\mathbf{0}}\right\rangle$ be a TCPN system. Consider some $E_{i}^{+}$such that $E_{i}^{+} \cap \operatorname{int}\left(\Re_{i}\right) \neq \emptyset$ and let $\mathbf{G}_{i}$ be a generator of it. Consider the matrix function $\operatorname{Cont}^{k}(\mathbf{A}, \mathbf{B})=$ $\left[\mathbf{B}, \mathbf{A B}, \ldots \mathbf{A}^{k} \mathbf{B}\right]$. Then:

1) If $\exists \mathbf{X}$ such that $C o n t^{|P|-1}\left(\mathbf{C} \boldsymbol{\Lambda} \boldsymbol{\Pi}_{i}, \mathbf{C}_{c f}^{i}\right) \cdot \mathbf{X}=\mathbf{G}_{i}$, then the system is controllable over $E_{i}^{+}$. Furthermore, if $T_{c f}^{i}=T_{c}$ then it is also a necessary condition for controllability over $E_{i}^{+}$, considering all the marking trajectories in $\Re_{i}$.

2) If $\nexists \mathbf{X}$ such that $C o n t^{|P|-1}\left(\mathbf{C} \boldsymbol{\Lambda} \boldsymbol{\Pi}_{i}, \mathbf{C}_{c}\right) \cdot \mathbf{X}=\mathbf{G}_{i}$, then the system is not controllable over $E_{i}^{+}$, considering all marking trajectories in $\Re_{i}$. 
Finally, next proposition (proved in [10]) introduces sufficient conditions for controllability over the union of sets of equilibrium markings of different regions.

Proposition 1: Let $\left\langle\mathcal{N}, \boldsymbol{\lambda}, \mathbf{m}_{\mathbf{0}}\right\rangle$ be a TCPN system. Consider equilibrium sets $E_{1}^{+}, E_{2}^{+}, \ldots, E_{j}^{+}$as defined above. If the system is BIC over each one and their union (i.e. $\bigcup_{i=1}^{j} E_{i}^{+}$) is connected, then the system is BIC over the union.

\section{Controlling Inside One Region}

In this section, a control law structure is proposed for the case in which the initial and desired markings, denoted as $\mathbf{m}_{0}$ and $\mathbf{m}_{d}$ respectively, belong to a common region $\Re_{i}$ and both are equilibrium markings.

In the sequel it is assumed that the system fulfills the sufficient condition for controllability of Corollary 1 (i.e. $\exists \mathbf{X}$ s.t. Cont $\left.^{|P|-1}\left(\mathbf{C} \Lambda \mathbf{\Pi}_{i}, \mathbf{C}_{c f}^{i}\right) \mathbf{X}=\mathbf{G}_{i}\right)$, so the system is controllable over $E_{i}^{+}$using only fully controllable transitions $T_{c f}^{i}$. According to this, control will be applied only to these transitions, while other controllable ones (those in $T_{c p}^{i}$ ) will be considered as uncontrollable.

Now, the classical feedback control law (for instance see [16]) is given by

$$
\mathbf{u}=\mathbf{u}_{d}+\mathbf{K e}
$$

where $\mathbf{K}$ is a gain matrix, $\mathbf{u}_{d}$ is the equilibrium input related to the desired marking $\mathbf{m}_{d}$ and $\mathbf{e}=\mathbf{m}-\mathbf{m}_{d}$ is the marking error.

Under these assumptions, if a negative and unbounded input could be applied to the system then $\mathbf{m}_{d}$ would be reachable by means of a control law like (2), but this is not the case because in $T C P N$ systems the input must be suitably bounded in order to be applied. However, as it will be proved next, given a stabilizing feedback gain matrix $\mathbf{K}$ it is possible to compute an intermediate desired marking $\mathbf{m}_{d}^{\prime}$, in the segment defined by $\mathbf{m}_{0}$ and $\mathbf{m}_{d}$ but close enough to $\mathbf{m}_{0}$, s.t. the input thus defined is suitably bounded along the trajectory, and this one lies in $\Re_{i}$. Computing several of these intermediate markings, $\mathbf{m}_{d}$ can be reached by jumping through them. Based on this idea, a control procedure is proposed, whose effectiveness and feasibility are formally proved through this section, but before that, let us introduce some useful notation.

Let $\mathbf{u}_{0}$ and $\mathbf{u}_{d}$ ( $\mathbf{w}_{0}$ and $\mathbf{w}_{d}$ ) be the equilibrium inputs (equilibrium flows) of $\mathbf{m}_{0}$ and $\mathbf{m}_{d}$, respectively ( $\mathbf{m}_{0}$ and $\mathbf{m}_{d}$ are equilibrium markings). Define the constant vectors $\mathbf{e}_{0}=\mathbf{m}_{0}-\mathbf{m}_{d}$ and $\Delta \mathbf{u}=\mathbf{u}_{0}-\mathbf{u}_{d}$, and consider the error $\mathbf{e}=\mathbf{m}-\mathbf{m}_{d}$. Consider a given intermediate target marking $\mathbf{m}_{d}^{\prime}$ in the segment defined by $\mathbf{m}_{0}$ and $\mathbf{m}_{d}$. This marking can be expressed as $\mathbf{m}_{d}^{\prime}=\mathbf{m}_{d}+\beta \mathbf{e}_{0}$, where $\beta$ is a scalar that belongs to $[0,1]$. By linearity, $\mathbf{m}_{d}^{\prime}$ is an equilibrium marking and one of its equilibrium inputs is given by $\mathbf{u}_{d}^{\prime}=\mathbf{u}_{d}+\beta \Delta \mathbf{u}$. The equilibrium flow of $\mathbf{m}_{d}^{\prime}$ and the error defined from it are given by $\mathbf{e}^{\prime}=\mathbf{m}-\mathbf{m}_{d}^{\prime}=\mathbf{e}-\beta \mathbf{e}_{0}$ and $\mathbf{w}_{d}^{\prime}=\mathbf{w}_{d}+\beta\left(\mathbf{w}_{0}-\right.$ $\left.\mathbf{w}_{d}\right)=\Lambda \boldsymbol{\Pi}_{i} \mathbf{m}_{d}^{\prime}-\mathbf{u}_{d}^{\prime}$, respectively.

\section{Control procedure:}

1) Compute a stabilizing feedback gain matrix $\mathbf{K}$ s.t. the controllable eigenvalues of the closed-loop state matrix $\left(\mathbf{C} \Lambda \mathbf{\Pi}_{i}-\mathbf{C K}\right)$ are different, real and negative, and the rows of $\mathbf{K}$, non related with transitions in $T_{c f}^{i}$, are null.

2) Given $\mathbf{m}_{0}$ an intermediate desired marking $\mathbf{m}_{d}^{\prime}$, which belongs to the segment defined by $\mathbf{m}_{0}$ and $\mathbf{m}_{d}$, is computed as follows

$$
\mathbf{m}_{d}^{\prime}=\mathbf{m}_{d}+\beta \mathbf{e}_{0}
$$

where $\beta$ is obtained by solving the following LPP

$$
\begin{aligned}
& \beta=\min \gamma \quad \text { subject to } \\
& \mathfrak{c}_{1}+\operatorname{neg}(\mathbf{A}) \mathbf{T}_{i}^{-1} \mathbf{e}+\gamma\left(\mathfrak{c}_{2}-\operatorname{neg}(\mathbf{A}) \mathbf{T}_{i}^{-1} \mathbf{e}_{0}\right) \geq \mathbf{0} \\
& \gamma \geq 0
\end{aligned}
$$

$\mathbf{T}_{i}$ is a similarity transformation matrix s.t. the transformed error $\mathbf{T}_{i}^{-1} \mathbf{e}$ is nonnegative and decreasing in the closed-loop system (i.e. $\dot{\mathbf{e}}=\left[\mathbf{C} \Lambda \boldsymbol{\Pi}_{i}-\mathbf{C K}\right] \mathbf{e}$ ), the function $n e g(A)$ is defined by elements as

$$
n e g(\mathbf{A})_{i, j}=\left\{\begin{array}{cc}
A_{i j} & \text { if } A_{i j}<0 \\
0 & \text { otherwise }
\end{array}\right.
$$

and the constants are defined as

$$
\begin{aligned}
\mathfrak{c}_{1}= & {\left[\begin{array}{c}
\mathbf{u}_{d} \\
\mathbf{w}_{d} \\
\left(\boldsymbol{\Pi}_{j}^{1}-\boldsymbol{\Pi}_{i}\right) \mathbf{m}_{d} \\
\vdots \\
\left(\boldsymbol{\Pi}_{j}^{r}-\boldsymbol{\Pi}_{i}\right) \mathbf{m}_{d}
\end{array}\right] \mathbf{A}=\left[\begin{array}{c}
\mathbf{K} \\
\Delta \boldsymbol{\Pi}_{i}-\mathbf{K} \\
\mathbf{\Pi}_{j}^{1}-\mathbf{\Pi}_{i} \\
\vdots \\
\mathbf{\Pi}_{j}^{r}-\mathbf{\Pi}_{i}
\end{array}\right] \mathbf{T}_{i} } \\
\mathfrak{c}_{2}= & {\left[\begin{array}{c}
\mathbf{w}_{0}-\mathbf{w}_{d} \\
\left(\boldsymbol{\Pi}_{j}^{1}-\mathbf{\Pi}_{i}\right) \mathbf{e}_{0} \\
\vdots \\
\left(\boldsymbol{\Pi}_{j}^{r}-\boldsymbol{\Pi}_{i}\right) \mathbf{e}_{0}
\end{array}\right] }
\end{aligned}
$$

considering all the configurations $\Pi_{j}^{1}, \ldots, \boldsymbol{\Pi}_{j}^{r}$ whose corresponding regions $\Re_{j}^{1}, \ldots, \Re_{j}^{r}$ are neighbors of $\Re_{i}$. Roughly speaking, first constant terms $\left(\mathbf{u}_{d}, \mathbf{K}\right.$ and $\left.\Delta \mathbf{u}\right)$ ensure that $\mathbf{u}>\mathbf{0}$, second terms ensure that $\mathbf{w}>\mathbf{0}$ and last terms guarantee that the trajectory will lie in $\Re_{i}$.

3) By means of the application of

$$
\mathbf{u}=\mathbf{u}_{d}^{\prime}+\mathbf{K}\left(\mathbf{m}-\mathbf{m}_{d}^{\prime}\right)
$$

the system is transferred towards $\mathbf{m}_{d}^{\prime}$ and the input is s.b. along the trajectory, which lies in $\Re_{i}$.

4) At any posterior time, a new intermediate desired marking $\mathbf{m}_{d}^{\prime}$ is computed, closer to $\mathbf{m}_{d}$ than the previous one, by solving again the LPP (4) (it is not required to reach the previous $\mathbf{m}_{d}^{\prime}$ in order to compute the new one). Then, the system is driven towards this new intermediate marking by means of the application of the corresponding control law (7), which is s.b. along the trajectory and this one lies in $\Re_{i}$. 
5) Iterating the previous step, the intermediate desired marking will be eventually computed as $\mathbf{m}_{d}$ (i.e. $\mathbf{m}_{d}^{\prime}=$ $\mathbf{m}_{d}$ ), which means that the system will asymptotically reach $\mathbf{m}_{d}$.

In order to prove the effectiveness of this control approach, we proceed as follows: in Proposition 2 the existence of $\mathbf{K}$ and $\mathbf{T}_{i}$, as they were previously defined, is proved. In Proposition 3 it is proved that the LPP (4) actually provides the next intermediate target marking, i.e. that the corresponding $\mathbf{m}_{d}^{\prime}$ is reached by means of (7) and this input is s.b.. Finally, in Proposition 4 it is proved that this LPP has always a solution, and this converges to $\beta=0$ (i.e. $\mathbf{m}_{d}^{\prime}=\mathbf{m}_{d}$ ).

Proposition 2: Consider a given $\mathbf{m}_{d}^{\prime} \in \operatorname{int}\left(\Re_{i}\right)$. The controllable poles of the closed-loop system can be arbitrarily assigned by means of a proper gain matrix $\mathbf{K}$, whose rows non related to transitions of $T_{c f}^{i}$ are null. If such poles are real, different and negative then the marking $\mathbf{m}_{d}^{\prime}$ is locally asymptotically stable in the closed-loop system. Moreover, there exists a similarity transformation $\mathbf{T}_{i}$ s.t. the transformed error $\mathbf{T}_{i}^{-1} \mathbf{e}^{\prime}$ is nonnegative and decreasing.

Proof: Denoting as $\mathbf{K}_{c f}^{i}$ the matrix built with the rows of $\mathbf{K}$ related to the transitions in $T_{c f}^{i}$, then $\mathbf{C K}=\mathbf{C}_{c f}^{i} \mathbf{K}_{c f}^{i}$ and $\mathbf{K}_{c f}^{i}$ fully determines $\mathbf{K}$. Applying the control law expressed in (7), the following closed-loop state equation is obtained

$$
\stackrel{\bullet}{\mathbf{e}^{\prime}}=\left(\mathbf{m}-\mathbf{m}_{d}^{\prime}\right)=\left[\mathbf{C} \Lambda \boldsymbol{\Pi}_{i}-\mathbf{C}_{c f}^{i} \mathbf{K}_{c f}^{i}\right] \mathbf{e}^{\prime}
$$

Consider the Kalman decomposition $\mathbf{T}_{k a l}$ (see, for instance, [16]), so

$$
\mathbf{T}_{k a l}^{-1} \mathbf{C} \Lambda \boldsymbol{\Pi}_{i} \mathbf{T}_{k a l}=\left[\begin{array}{cc}
\mathbf{A}_{11} & \mathbf{A}_{12} \\
\mathbf{0} & \mathbf{A}_{22}
\end{array}\right], \mathbf{T}_{k a l}^{-1} \mathbf{C}_{c f}^{i}=\left[\begin{array}{c}
\mathbf{B} \\
\mathbf{0}
\end{array}\right]
$$

and the pair $\left(\mathbf{A}_{11}, \mathbf{B}\right)$ is controllable in the classical sense. Then, the eigenvalues of $\left(\mathbf{A}_{11}-\mathbf{B} \mathbf{K}_{k a l}\right)$ can be arbitrarily assigned by a proper choice of a gain matrix $\mathbf{K}_{k a l}$. Assume that $\mathbf{K}_{k a l}$ places those eigenvalues as real, different and negative. Consider the gain matrix of the original system s.t. $\mathbf{K}_{c f}^{i}=\left[\begin{array}{ll}\mathbf{K}_{k a l} & \mathbf{0}\end{array}\right] \mathbf{T}_{k a l}^{-1}$, so the controllable eigenvalues of $\left[\mathbf{C} \Lambda \boldsymbol{\Pi}_{i}-\mathbf{C K}\right]$ are equal to those assigned by $\mathbf{K}_{k a l}$. The transformed closed-loop system is given by

$\dot{\epsilon^{\prime}}=\mathbf{T}_{k a l}^{-1} \dot{\mathbf{e}}^{\prime}=\left[\begin{array}{cc}\left(\mathbf{A}_{11}-\mathbf{B} \mathbf{K}_{k a l}\right) & \mathbf{A}_{12} \\ \mathbf{0} & \mathbf{A}_{22}\end{array}\right] \epsilon^{\prime}, \epsilon_{0}^{\prime}=\left[\begin{array}{c}\epsilon_{10}^{\prime} \\ \epsilon_{20}^{\prime}\end{array}\right]$

where $\epsilon_{0}^{\prime}=\mathbf{T}_{k a l}^{-1} \mathbf{e}_{0}^{\prime}$. Now, by controllability hypothesis $\mathbf{G}_{i} \in \operatorname{Span}\left\{C_{\text {Cont }}^{|P|-1}\left(\mathbf{C} \Lambda \mathbf{\Pi}_{i}, \mathbf{C}_{c f}^{i}\right)\right\}$, so $\mathbf{T}_{k a l}^{-1} \mathbf{G}_{i} \in$ $\operatorname{Span}\left\{\right.$ Cont $\left.^{|P|-1}\left(\mathbf{T}_{k a l}^{-1} \mathbf{C} \Lambda \mathbf{\Pi}_{i} \mathbf{T}_{k a l}, \mathbf{T}_{k a l}^{-1} \mathbf{C}_{c f}^{i}\right)\right\}$, which is equivalent to

$$
\mathbf{T}_{k a l}^{-1} \mathbf{G}_{i} \in \operatorname{Span}\left\{\left[\begin{array}{cccc}
\mathbf{B} & \mathbf{A}_{11} \mathbf{B} & , \ldots, & \mathbf{A}_{11}^{|P|-1} \mathbf{B} \\
\mathbf{0} & \mathbf{0} & , \ldots, & \mathbf{0}
\end{array}\right]\right\}
$$

Since $\mathbf{T}_{k a l}^{-1} \mathbf{e}_{0}^{\prime} \in \operatorname{Span}\left\{\mathbf{T}_{k a l}^{-1} \mathbf{G}_{i}\right\}$ then $\epsilon_{20}^{\prime}=\mathbf{0}$, i.e. the transformed initial error is null at the uncontrollable part. Now, consider a modal decomposition of $\left(\mathbf{A}_{11}-\mathbf{B} \mathbf{K}_{k a l}\right)$, i.e. a modal matrix $\mathbf{V}$ s.t. $\mathbf{V}^{-1}\left(\mathbf{A}_{11}-\mathbf{B} \mathbf{K}_{k a l}\right) \mathbf{V}=\mathbf{D}$ where $\mathbf{D}$ is diagonal, and compute a diagonal matrix $\mathbf{S}$ whose elements are in $\{-1,1\}$ (so $\mathbf{S}=\mathbf{S}^{-1}$ ) in such a way that $\mathbf{S} \mathbf{V}^{-1} \epsilon_{10}^{\prime} \geq$ 0. Then, defining the similarity transformation

$$
\mathbf{T}_{i}=\mathbf{T}_{k a l}\left[\begin{array}{cc}
\mathbf{V S} & \mathbf{0} \\
\mathbf{0} & \mathbf{I}
\end{array}\right]
$$

the original closed-loop system (8) can be transformed as

$$
\begin{aligned}
& \mathbf{T}_{i}^{-1} \dot{\mathbf{e}^{\prime}}=\left[\begin{array}{l}
\varepsilon_{1}^{\prime} \\
\varepsilon_{2}^{\prime}
\end{array}\right]=\left[\begin{array}{ll}
\mathbf{D} & (\mathbf{V S})^{-1} \mathbf{A}_{12} \\
\mathbf{0} & \mathbf{A}_{22}
\end{array}\right]\left[\begin{array}{l}
\varepsilon_{1}^{\prime} \\
\varepsilon_{2}^{\prime}
\end{array}\right] \\
& \mathbf{T}_{i}^{-1} \mathbf{e}_{0}^{\prime}=\varepsilon_{0}^{\prime}=\left[\begin{array}{l}
\varepsilon_{10}^{\prime} \\
\varepsilon_{20}^{\prime}
\end{array}\right] \geq \mathbf{0}
\end{aligned}
$$

Since $\varepsilon_{20}^{\prime}=\epsilon_{20}^{\prime}=\mathbf{0}$, the solution of such state equation is given by

$$
\begin{aligned}
& \varepsilon_{1}^{\prime}(\tau)=e^{\mathbf{D} \tau} \varepsilon_{10}^{\prime} \\
& \varepsilon_{2}^{\prime}(\tau)=\varepsilon_{20}^{\prime}=\mathbf{0}
\end{aligned}
$$

Therefore, $\varepsilon_{0}^{\prime} \geq \varepsilon^{\prime}(\tau) \geq \mathbf{0} \forall \tau$, and the error converges to zero.

In the sequel, let us assume that $\mathbf{K}$ is s.t. the controllable poles of the closed-loop system are real, different and negative.

Proposition 3: Consider the initial and desired markings $\mathbf{m}_{0}, \mathbf{m}_{d} \in \operatorname{int}\left\{\Re_{i}\right\}$. Suppose that at some time $\tau_{1}, \mathbf{m}\left(\tau_{1}\right) \in$ $\operatorname{int}\left\{\Re_{i}\right\}$ and a solution $\beta$ for the LPP (4) is computed. Consider the intermediate desired marking as in (3). If the input (7) is being applied then $\mathbf{m}_{d}^{\prime}$ will be reached through a trajectory inside $\Re_{i}$. Moreover, such input will be suitable bounded along the marking trajectory.

Proof: According to (4) and denoting $\mathbf{e}^{\prime}\left(\tau_{1}\right)=\mathbf{e}\left(\tau_{1}\right)-$ $\beta \mathbf{e}_{0}$

$$
\mathfrak{c}_{1}+\beta \mathfrak{c}_{2}+\operatorname{neg}(\mathbf{A}) \mathbf{T}_{i}^{-1} \mathbf{e}^{\prime}\left(\tau_{1}\right) \geq \mathbf{0}
$$

Now, since the control law (7) is being applied, according to Proposition 2 the error of the closed-loop system fulfills $\mathbf{T}_{i}^{-1} \mathbf{e}^{\prime}\left(\tau_{1}\right) \geq \mathbf{T}_{i}^{-1} \mathbf{e}^{\prime}(\tau) \geq \mathbf{0}$ for $\tau \geq$ $\tau_{1}$, so, $\operatorname{neg}(\mathbf{A}) \mathbf{T}_{i}^{-1} \mathbf{e}^{\prime}\left(\tau_{1}\right) \leq \operatorname{neg}(\mathbf{A}) \mathbf{T}_{i}^{-1} \mathbf{e}^{\prime}(\tau)$ (because $n e g(\mathbf{A}) \leq \mathbf{0}$ by definition), furthermore, since $\mathbf{T}_{i}^{-1} \mathbf{e}^{\prime}(\tau) \geq$ $\mathbf{0}$, then $n e g(\mathbf{A}) \mathbf{T}_{i}^{-1} \mathbf{e}^{\prime}(\tau) \leq \mathbf{A T}_{i}^{-1} \mathbf{e}^{\prime}(\tau)$. Therefore $n e g(\mathbf{A}) \mathbf{T}_{i}^{-1} \mathbf{e}^{\prime}\left(\tau_{1}\right) \leq \mathbf{A} \mathbf{T}_{i}^{-1} \mathbf{e}^{\prime}(\tau)$, so, substituting into (11) we obtain

$$
\begin{gathered}
\mathfrak{c}_{1}+\beta \mathfrak{c}_{2}+\mathbf{A T}_{i}^{-1} \mathbf{e}^{\prime}(\tau) \geq \mathbf{0} \\
\forall \tau \geq \tau_{1}
\end{gathered}
$$

Substituting the constants (6) and using the definitions of $\mathbf{e}^{\prime}, \mathbf{w}_{d}^{\prime}$ and $\mathbf{m}_{d}^{\prime}$, it can be demonstrated that previous inequalities are equivalent to

$$
\begin{gathered}
\mathbf{u}(\tau) \geq \mathbf{0} \\
\Lambda \boldsymbol{\Pi}_{i} \mathbf{m}(\tau)-\mathbf{u}(\tau) \geq \mathbf{0} \\
\left(\boldsymbol{\Pi}_{j}^{1}-\boldsymbol{\Pi}_{i}\right) \mathbf{m}(\tau) \geq \mathbf{0} \\
\vdots \\
\left(\boldsymbol{\Pi}_{j}^{r}-\boldsymbol{\Pi}_{i}\right) \mathbf{m}(\tau) \geq \mathbf{0} \\
\forall \tau \geq \tau_{1}
\end{gathered}
$$

Therefore, $\mathbf{u}(\tau)$ is s.b. for all future time and the system lies in $\Re_{i}$.

The LPP (4) can be solved very efficiently on-line, because it can be reduced to the problem of finding the minimum 
entry of some computed vector. Furthermore, some of the inequalities are always fulfilled and can be eliminated (those involving uncontrollable transitions and null constants).

Proposition 4: Suppose that the initial and desired markings $\left(\mathbf{m}_{0}\right.$ and $\left.\mathbf{m}_{d}\right)$ belong to $\operatorname{int}\left(\Re_{i}\right) \cap E_{i}^{+}$, and that the entries of $\mathbf{u}_{0}$ and $\mathbf{u}_{d}$ are positive for all the fully controllable transitions. Then, there exists solution $\beta$ for the LPP (4) at $\mathbf{m}_{0}$. Moreover, if the control procedure previously introduced is being applied then there will exist solution $\beta$ at all future markings. Furthermore, the closer the marking is to $\mathbf{m}_{d}$, the lower is this solution, until obtaining a value of 0 .

Proof: Consider the inequality of (4) but reorder it as

$$
\mathfrak{c}_{1}+\gamma \mathfrak{c}_{2}+\operatorname{neg}(\mathbf{A}) \mathbf{T}_{i}^{-1}\left(\mathbf{e}-\gamma \mathbf{e}_{0}\right) \geq \mathbf{0}
$$

First consider a given $\gamma \in[0,1]$. In this way, the marking $\mathbf{m}_{d}^{\prime}=\mathbf{m}_{d}+\gamma \mathbf{e}_{0}$ is in the segment defined by $\mathbf{m}_{0}$ and $\mathbf{m}_{d}$, so, it belongs to $\operatorname{int}\left(\Re_{i}\right)$ and the vector

$$
\mathfrak{c}_{1}+\gamma \mathfrak{c}_{2}=\left[\begin{array}{c}
\mathbf{u}_{d}^{\prime} \\
\mathbf{w}_{d}^{\prime} \\
\left(\boldsymbol{\Pi}_{j}^{1}-\mathbf{\Pi}_{i}\right) \mathbf{m}_{d}^{\prime} \\
\vdots \\
\left(\mathbf{\Pi}_{j}^{r}-\boldsymbol{\Pi}_{i}\right) \mathbf{m}_{d}^{\prime}
\end{array}\right]
$$

is positive at the relevant entries (those that correspond to non null rows of $\mathbf{A}$ ). Therefore, for a small enough value of $\left(\mathbf{e}-\gamma \mathbf{e}_{0}\right)=\mathbf{e}^{\prime}$, i.e. for $\mathbf{m}$ close enough to $\mathbf{m}_{d}^{\prime}, \gamma$ is a solution for (12). A particular case occurs at $\mathbf{m}_{0}$, in which $\left(\mathbf{e}-\gamma \mathbf{e}_{0}\right)=(1-\gamma) \mathbf{e}_{0}=\mathbf{e}^{\prime}$ (i.e. a value of $\gamma$ close enough to 1 makes $\mathbf{e}^{\prime}$ be small enough, and then such $\gamma$ is a solution for (12) and the LPP).

Now, suppose that at time $\tau_{1}$ a solution $\beta_{1}$ is computed. Substituting $\gamma=\beta_{1}-\Delta \gamma$ into (12) and reordering the terms we obtain

$$
\begin{aligned}
\mathfrak{c}_{1}+\beta_{1} \mathfrak{c}_{2}+ & \operatorname{neg}(\mathbf{A}) \mathbf{T}_{i}^{-1}\left(\mathbf{e}-\beta_{1} \mathbf{e}_{0}\right)- \\
& \Delta \gamma\left[\mathfrak{c}_{2}-\operatorname{neg}(\mathbf{A}) \mathbf{T}_{i}^{-1} \mathbf{e}_{\mathbf{0}}\right] \geq \mathbf{0}
\end{aligned}
$$

Consider a future time $\tau_{2}>\tau_{1}$. If the control law (7) is being applied (with $\beta=\beta_{1}$ ) then $\mathbf{T}_{i}^{-1} \mathbf{e}^{\prime}\left(\tau_{2}\right) \leq \mathbf{T}_{i}^{-1} \mathbf{e}^{\prime}\left(\tau_{1}\right)$, which implies that neg $(\mathbf{A}) \mathbf{T}_{i}^{-1} \mathbf{e}^{\prime}\left(\tau_{2}\right) \geq n e g(\mathbf{A}) \mathbf{T}_{i}^{-1} \mathbf{e}^{\prime}\left(\tau_{1}\right)$, so, at $\tau_{2}$ there will exist $\Delta \gamma>0$ that fulfills (13), i.e. as $\mathbf{m}$ approximates to the intermediate marking a lower solution is obtained.

Finally, notice that a lower solution $\beta_{2}$ cannot be obtained only if either the current one is null (i.e. $\beta_{1}=0$ ) or it fulfills that $\mathfrak{c}_{1}+\beta_{1} \mathfrak{c}_{2}$ has null elements at the relevant entries. However, this last condition implies that $\mathbf{m}_{d}^{\prime}=\mathbf{m}_{d}+\beta_{1} \mathbf{e}_{0}$ belongs to the frontier of $\Re_{i}$ and/or there is a null entry of $\mathbf{u}_{d}^{\prime}$ related to a transition in $T_{c f}^{i}$, but under the hypothesis, it never occurs. Therefore, the solution $\beta=0$ will be eventually obtained.

The computation of $\beta$ can be done at every sampling or just at some of them. Moreover, any pole assignment technique can be used for computing $\mathbf{K}$. It can be proved that this control approach can also be applied in the cases in which $\mathbf{m}_{d}$ or $\mathbf{m}_{0}$ belongs to the frontier of the region, and/or $\mathbf{u}_{d}$ or $\mathbf{u}_{0}$ have null entries related to fully controllable transitions.

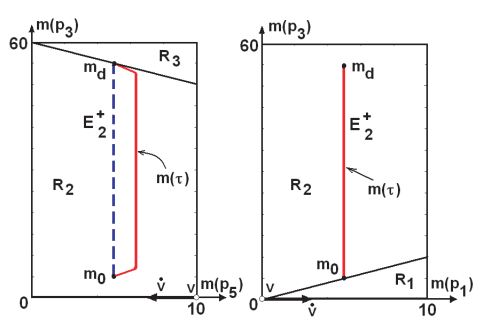

(b)

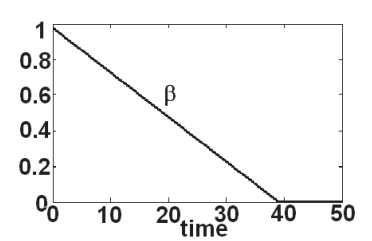

(c)

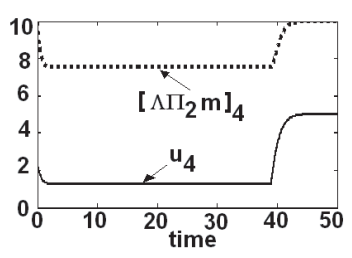

(d)
Fig. 2. a) TCPN system controllable over $E_{2}^{+}$. b) Projections of Class $\left(\mathbf{m}_{0}\right)$ on the planes $\left[\mathbf{m}\left(p_{5}\right), \mathbf{m}\left(p_{3}\right)\right]$ and $\left[\mathbf{m}\left(p_{1}\right), \mathbf{m}\left(p_{3}\right)\right]$, the dashed line is $E_{2}^{+}$while the continuous one is the trajectory of the closedloop system (both coincide in the second projection)). c) Computed value for $\beta, \mathrm{d})$ input signal $\mathbf{u}$ and flow $\left(\Lambda \Pi_{2} \mathbf{m}\right)$ of $t_{4}$ during the closed-loop evolution.

Example 2: Consider the system of fig. 2(a) with $\lambda_{1}=$ $\lambda_{2}=\lambda_{3}=1$ and $\lambda_{4}=2$. Let $T_{c}=\left\{t_{4}\right\}$. This system has two synchronizations and only 3 feasible regions (there would be 4 but $p_{3}$ and $p_{4}$ cannot constraint their output transitions simultaneously). Projections of $\operatorname{Class}\left(\mathbf{m}_{0}\right)$ on the planes $\left[\mathbf{m}\left(p_{5}\right), \mathbf{m}\left(p_{3}\right)\right]$ and $\left[\mathbf{m}\left(p_{1}\right), \mathbf{m}\left(p_{3}\right)\right]$ are shown in fig. 2(b) (the marking of only three places is required to defined a reachable marking, then two projections are sufficient for representing $\operatorname{Class}\left(\mathbf{m}_{0}\right)$ ). The initial marking is $\mathbf{m}_{0}=[5,5,5,55,5,5]^{T}$ while the desired one is $\mathbf{m}_{d}=[5,5,55,5,5,5]^{T}$. Both markings belong to the same region $\Re_{2}$.

First, we will show, through this example, that the control problem of TCPN systems is not trivial. Suppose that we would like to apply a classical feedback control law (2), then we would find that the rank of the controllability matrix for this configuration is 2 , so the system is not controllable in the classical sense and it could be impossible to reach $\mathbf{m}_{d}$ according to the classical theory. However, the uncontrollable poles are stable, at least it is possible to stabilize the system, so we compute a feedback gain matrix $\mathbf{K}$, that places the controllable poles of the closed-loop system at -1 and -2 . With such control law, the input at the initial marking is computed as $u_{4}=-95$, which cannot be applied. Even when we can computed another gain matrices and eventually we could obtain a positive value at the initial marking, it would not guarantee the input to be suitable bounded along the whole trajectory (not only at the initial marking), and that this one would lie in $\Re_{2}$. Following a different approach, let us try to apply the techniques proposed in [11]. In such case, we need to compute values for the input at the vertices of $\Re_{2}$ that direct the field vector into the same region. The problem in our system is that for some vertices the input must be null. That occurs for the vertex $\mathbf{v}=[0,10,0,60,10,0]^{T}$ drawn 
in fig. 2(b), therefore, for all the admissible inputs the field vector is given by $\dot{\mathbf{v}}=\mathbf{C} \boldsymbol{\Lambda} \boldsymbol{\Pi}_{2} \mathbf{v}=[-10,10,0,0,-10,10]^{T}$. It can be seen in fig. 2(b) that $\dot{v}$ is directed towards $\Re_{1}$ (vector $\dot{v}$ is scaled in fig. 2(b)) then it is not possible to guarantee that the system will remain inside $\Re_{2}$ by using the results introduced in [11] and so, the control law cannot be computed. This kind of problem occurs very frequently in $T C P N$ systems, since for all the transitions there exists a "facet" of the Class $\left(\mathbf{m}_{0}\right)$ in which its enabling degree is null, then the field vector cannot be directed at the corresponding vertices.

Now, let us come back to the techniques developed in this work. The set of equilibrium markings in $\Re_{2}$, i.e. $E_{2}^{+}$, is shown in fig. 2(b) as a dashed line. A generator for it is given by $\mathbf{G}_{2}=[0,0,1,-1,0,0]^{T}$. For this case, $T_{c}=T_{c f}^{2}$ and the system is controllable over $E_{2}^{+}$(according to Corollary 1). The control procedure, introduced through this section, was applied to this system. For that, we considered the same feedback gain matrix $\mathbf{K}$, that places the controllable poles of the closed-loop system at -1 and -2 , and also, we computed the required similarity transformation matrix $\mathbf{T}_{2}$. The LPP was continuously solved (at each simulation time step). Fig. 2 (c) shows the evolution of the value computed for $\beta$. The closed-loop trajectory is drawn in fig. 2(b) (continuous line). It can be seen that this control law successfully transfer the state from $\mathbf{m}_{0}$ to $\mathbf{m}_{d}$ through a trajectory in $\Re_{2}$, moreover, it can be seen in fig. 2(d) that the input is s.b..

\section{Controlling Between Regions}

This section is devoted to advance the ideas leading to the generalization of the previously introduced control law structure. In our experience, it always has been found (but still not proved) that set $E_{q} S$ is connected. In such case, a generalization of the control law for different regions is reduced to the problem of transferring the system from an equilibrium marking $\mathbf{m}_{01} \in \Re_{1}$ to another one $\mathbf{m}_{d 2} \in \Re_{2}$, where $\Re_{1}$ and $\Re_{2}$ are neighbor regions, which is studied in this section. It is assumed in the sequel that the system fulfills the sufficient conditions for controllability over $E_{1}^{+}$ and $E_{2}^{+}$of Corollary 1. Also, it is assumed that there exists an equilibrium marking $\mathbf{m}_{\text {int }}$ that belongs to $E_{1}^{+} \cap E_{2}^{+}$but not to another region (if $E_{q} S$ is connected then $\exists \mathbf{m}_{\text {int }} \in$ $E_{1} \cap E_{2}$ ).

Under the established hypothesis, a control law for each region can be computed by using the control law scheme introduced before. In this way, a first idea is to compute and apply a control law that transfers the marking from $\mathbf{m}_{01} \in \Re_{1}$ to $\mathbf{m}_{\text {int }}$, through a trajectory inside $\Re_{1}$, and another control law that transfers the marking from $\mathbf{m}_{\text {int }}$ to $\mathbf{m}_{d 2} \in \Re_{2}$, through a trajectory inside $\Re_{2}$. However, the first control law will transfer the marking towards $\mathbf{m}_{\text {int }}$ in infinite time, so the second control law will never be applied. Then, the problem can be reduced to the computation of a control law that transfers the marking from any $\mathbf{m} \in \Re_{1}$ in a neighborhood of $\mathbf{m}_{\text {int }}$, to an equilibrium marking $\mathbf{m}_{d 2}^{\prime} \in$ $\Re_{2}-\Re_{1}$. Such control law exists because the system fulfills the conditions of Proposition 1.
In order to solve this problem, consider the LPP (4) for $\Re_{1}$ with $\mathbf{m}_{\text {int }}$ as the desired marking, $\Delta \mathbf{u}=\mathbf{u}_{01}-\mathbf{u}_{\text {int }}$ and without the inequalities $\gamma \geq 0$ and that which involves $\Pi_{2}-\Pi_{1}$. Then, this new LPP is obtained

$$
\begin{aligned}
\beta= & \min \gamma \quad \text { subject to } \\
& \mathfrak{c}_{1}+\operatorname{neg}(\mathbf{A}) \mathbf{T}_{1}^{-1} \mathbf{e}+\gamma\left(\mathfrak{c}_{2}-\operatorname{neg}(\mathbf{A}) \mathbf{T}_{1}^{-1} \mathbf{e}_{0}\right) \geq \mathbf{0} \\
\mathfrak{c}_{1}= & {\left[\begin{array}{c}
\mathbf{0} \\
\mathbf{u}_{\text {int }} \\
\mathbf{w}_{\text {int }} \\
\left(\boldsymbol{\Pi}_{j}^{1}-\mathbf{\Pi}_{1}\right) \mathbf{m}_{\text {int }} \\
\vdots \\
\left(\boldsymbol{\Pi}_{j}^{r}-\mathbf{\Pi}_{1}\right) \mathbf{m}_{\text {int }}
\end{array}\right], \mathbf{A}=\left[\begin{array}{c}
\mathbf{K} \\
\Lambda \boldsymbol{\Pi}_{1}-\mathbf{K}_{1} \\
\boldsymbol{\Pi}_{j}^{1}-\mathbf{\Pi}_{1} \\
\vdots \\
\mathbf{\Pi}_{j}^{r}-\mathbf{\Pi}_{1}
\end{array}\right] \mathbf{T}_{1} } \\
\mathfrak{c}_{2}= & {\left[\begin{array}{c}
\mathbf{w}_{01}-\mathbf{w}_{\text {int }} \\
\left(\boldsymbol{\Pi}_{j}^{1}-\boldsymbol{\Pi}_{1}\right) \mathbf{\Delta} \mathbf{u} \\
\vdots \\
\left(\boldsymbol{\Pi}_{j}^{r}-\mathbf{\Pi}_{1}\right) \mathbf{\Delta} \mathbf{u}
\end{array}\right] }
\end{aligned}
$$

where $\Pi_{j}^{1}, \ldots, \Pi_{j}^{r}$ are related to all the neighbor regions of $\Re_{1}$, excepting $\boldsymbol{\Pi}_{2}$.

Notice that, if at a given marking $\mathbf{m}\left(\tau_{1}\right)$, close enough to $\mathbf{m}_{\text {int }}$, a value of $\beta<0$ were computed, then the target marking $\mathbf{m}_{d}^{\prime}=\beta \mathbf{m}_{01}+(1-\beta) \mathbf{m}_{\text {int }}$ would belong to $\Re_{2}$. In such case, the control law (7) would transfer the system from $\mathbf{m}\left(\tau_{1}\right) \in \Re_{1}$ to some $\mathbf{m}\left(\tau_{2}\right) \in \Re_{2} \cap \Re_{1}$, and once the marking would reach the frontier, the previously introduced control procedure could be applied in order to finally reach $\mathbf{m}_{d 2} \in \Re_{2}$. It is easy to prove that a value $\beta<0$ can be computed if the entries of $\mathbf{u}_{i n t}$, related to transition of $T_{c f}^{1}$, are positive. Furthermore, it can be demonstrated that this property is fulfilled for several cases (for example, mono-Tsemiflow nets whose configurations "contain" P-flows).

Now, denote the solution for the modified LPP (14) as $\beta_{1}$ and the solution for the LPP (4) defined for $\Re_{2}$ as $\beta_{2}$. A simple way to guarantee that a solution $\beta_{2}<1$ will be computed once the system reach the common frontier, consists in computing both, $\beta_{1}$ and $\beta_{2}$, simultaneously when the marking is approximating to the frontier, and adding a new rule: if $\beta_{1}<0$ but $\beta_{2} \geq 1$ then consider $\beta_{1}$ as zero for the computation of $\mathbf{m}_{d}^{\prime}$. In this way, an input for crossing the border is computed (i.e. $\beta_{1}<0$ is considered) only if $\beta_{2}<1$, otherwise, the system will be transferred towards $\mathbf{m}_{\text {int }}$ and it will remain in $\Re_{1}$, until reaching a neighborhood of $\mathbf{m}_{\text {int }}$ in which such values can be computed (such neighborhood exists because $\beta_{2}<1$ and $\beta_{1}<0$ can be obtained at $\mathbf{m}_{\text {int }}$ ). Finally, the system will probably cross the frontier at a non equilibrium marking, then the error of the uncontrollable part (for the system at $\Re_{2}$ ) may not be null, in such case, we have to ask for the non controllable poles (non related to P-flows) to be stable.

Example 3: Consider the system of fig. 3(a) with $\boldsymbol{\Lambda}=$ I. Let $T_{c}=\left\{t_{1}\right\}$. This system has one synchronization, which leads to 2 regions that are denoted as $\Re_{1}$ (in which $p_{5}$ is constraining $t_{5}$ ) and $\Re_{2}$ (in which $p_{6}$ is constraining $t_{5}$ ). The initial marking is $\mathbf{m}_{0}=$ 


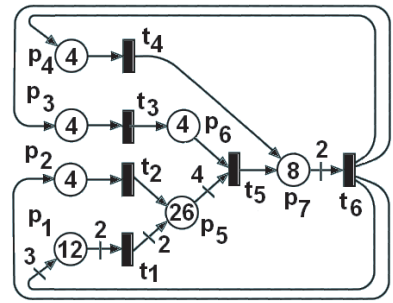

(a)

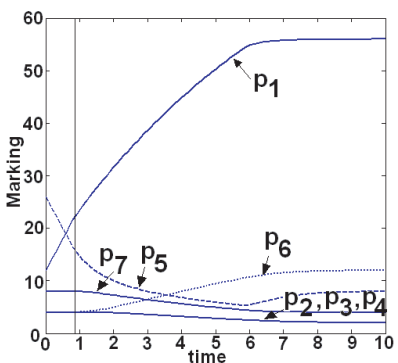

(b)
Fig. 3. a) TCPN system controllable over $E_{1}^{+} \cup E_{2}^{+}$. b) Marking of places for the trajectory described by the closed-loop system.

$[12,4,4,4,26,4,8]^{T}$ which belongs to $\Re_{2}$. Consider a desired marking $\mathbf{m}_{d}=[56,2,2,2,8,12,4]^{T}$, which belongs to $\Re_{1}$. There exists an interface equilibrium marking $\mathbf{m}_{\text {int }}=$ $[22,4,4,4,16,4,8]^{T}$ s.t. $\mathbf{m}_{\text {int }} \in E_{1}^{+} \cap E_{2}^{+}$. This system fulfills the conditions of Proposition 1 for the controllability over $E_{1}^{+} \cup E_{2}^{+}$. In this way, stabilizing gain matrices were computed for each region. The equilibrium input of the interface marking $\mathbf{m}_{\text {int }}$ is given by $\mathbf{u}_{\text {int }}=[5,0,0,0,0,0]^{T}$, i.e. it is positive at the entry related to $t_{1}$ (the only controllable transition), so, the control law approach introduced in this section can be used for transferring the system through the region's frontier. Applying this, the system is successfully transferred from $\mathbf{m}_{0}$ to $\mathbf{m}_{d}$ through a trajectory in which the input is s.b.. The markings of the places through this trajectory are shown in fig. 3(b), where the vertical line denotes the change of region (from $\Re_{2}$ to $\Re_{1}$ ).

\section{CONCLUSIONS}

This work addresses the computation of control laws for timed continuous Petri net $(T C P N)$ systems with uncontrollable transitions. The main contribution is the adaptation of the classical state feedback control law approach to these systems. The implementation of this control law consists in the computation of a suitable gain matrix for each region, and the resolution of a LPP during its application for computing suitable intermediate target markings that guarantees the boundedness of the input, obtaining thus a piecewise-linear constrained control structure. Moreover, it is proved that such control law can always be computed, and it transfers the marking towards the desired one, whenever the conditions for controllability are fulfilled (Corollary 1 and Proposition 1) and there exist suitable interface markings between the regions.

\section{REFERENCES}

[1] H. Alla, R. David, Continuous and hybrid Petri nets, Journal of Circuits, Systems, and Computers, vol. 8(1), 1998, pp 159-188.

[2] M. Silva, L. Recalde, L., Petri nets and integrality relaxations: A view of continuous Petri nets, IEEE Trans. on Systems, Man and Cybernetics, vol. 32(4), 2002, pp 314-327.

[3] C. Mahulea, L. Recalde, M. Silva, On performance monotonicity and basic servers semantics of continuous Petri nets, In: 8th Int. Workshop on Discrete Event Systems, Ann Arbor, USA, 2006, pp 345-351.

[4] E. Sotang, An algebraic approach to bounded controllability of linear systems, Int. Journal of Control, vol. 39, 1984, pp 181-188.

[5] H. Sussmann, E. Sontag, Y. Yang, A General Result on the Stabilization of Linear Systems using Bounded Controls, Proceedings of the 32nd Conference on Decisions and control, San Antonio, USA, 1993, pp 1802-1807.

[6] R. Brammer, Controllability in linear autonomous systems with positive controllers, SIAM Journal Control, vol. 10(2), 1972, pp 329-353.

[7] L. Rodrigues, E. Boukas, Piecewise-linear $H_{\infty}$ controller synthesis with applications to inventory control of switched production systems, Automatica, vol. 42(8), 2006, pp 1245-1254.

[8] A. Hassibi, S. Boyd, Quadratic stabilization and control of piecewiselinear systems, Proceedings of American Control Conference, Philadelphia, USA, vol. 6, 1998, pp 3659-3664.

[9] C. Mahulea, A. Ramírez-Trevino, L. Recalde, M. Silva, Steady state control reference and token conservation laws in continuous Petri net systems, IEEE Trans. on Automation Science and Engineering, vol. 2(5), 2008, pp 726-741.

[10] C.R. Vázquez, A. Ramírez-Trevino, L. Recalde, M. Silva, M., On controllability of timed continuous Petri nets, 11th Int. Workshop Hybrid Systems: Computational and Control, (Egerstedt M., Mishra B. eds.), Lecture Notes in Computer Sciences, Springer Verlag, vol. 4981, 2008, pp 528-541.

[11] L.C.G.J.M. Habets, J.H. van Schuppen, A control problem for affine dynamical systems on a full-dimensional polytope, Automatica, vol. 40, 2004, pp 21-35.

[12] C. Mahulea, A. Giua, L. Recalde, C. Seatzu, M. Silva, Optimal model predictive control of Timed Continuous Petri nets, IEEE Transsactions on Automatic Control, vol. 53(7), 2008, pp 1731-1735.

[13] J. Xu, L. Recalde, M. Silva, Tracking control of join-free timed continuous Petri net systems under infinite servers semantics, Discrete Event Dynamic Systems, vol. 18(2), 2008, pp 263-283.

[14] D. Lefebvre, D. Catherine, E. Leclerq, F. Druaux, Some contributions with petri nets for the modelling, analysis and control of HDS, Proccedings of the International Conference on Hybrid Systems and Applications, LA, USA, vol. 1(4), 2007, pp 451-465.

[15] J. Júlvez, L. Recalde, M. Silva, On reachability in autonomous continuous Petri net systems, Proc. of the $24^{\text {th }}$ Int. Conf. on Application and Theory of Petri Nets (van der Aalst, W., Best, E., eds.) Lecture Notes in Computer Sciences, Springer Verlag, vol. 2679, 2003, pp 221-240.

[16] C. Chen, Linear system theory and design, Oxford University Press USA, 1984. 